\title{
On the integration of reconfigurable intelligent surfaces in real-world environments
}

\author{
A. Díaz-Rubio, Senior Member, IEEE, S. Kosulnikov, and S.A. Tretyakov, Fellow, IEEE
}

\begin{abstract}
The use of reconfigurable intelligent surfaces (RISs) for optimization of propagation channels is one of the most promising and revolutionizing techniques for improving the efficiency of the next generation of communications systems. In this work, we combine the physical optics approximation and the theory of diffraction gratings to study the scattering properties of finite-size metasurfaces mounted on partially reflecting walls and illuminated by directive antennas. We consider both reflective and refractive metasurfaces designed to control both reflection and transmission of waves. We start the analysis under the assumption of uniform, plane-wave illumination, and then discuss non-uniform illuminations by directive antennas.
\end{abstract}

\section{INTRODUCTION}

Recently, many researchers have been studying potential improvements of wireless telecommunication systems with the use of reconfigurable intelligent metasurfaces (RIS) [1][9]. The governing idea is that making some parts of the propagation environment tunable and adjustable, one can optimize it together with the optimization of the transmitters and receivers. To realize it, it has been proposed to cover parts of room walls or building facades with engineered and tunable reflecting or refracting surfaces (metasurfaces). Metasurfaces are thin arrays of electrically small elements (unit cells, also called meta-atoms) that are made tunable, most commonly by incorporating varactors or switches into each meta-atom. By tuning the array elements, it is possible to direct scattered waves into the desired direction or focus at the desired spot, this way allowing optimization of propagation channels.

Obviously, such reflecting or refracting arrays of tunable elements remind us the notion of reflectarray and transmitarray antennas [10]-[12]. Indeed, there are significant similarities, and the knowledge of reflectarray theory and design is useful in work on reconfigurable metasurfaces. However, the notion of tunable metasurfaces is more general. While the distance between the elements in reflectarrays is typically half the wavelength, metasurfaces use advanced possibilities offered by subwavelength structuring of the array fabric. The reflectarrays function as phase-gradient arrays, and suffer from parasitic scattering when the desired reflection is significantly different from specular reflection [13]-[17]. In contrast, metasurfaces can offer theoretically perfect (assuming precise settings and

(Corresponding author: A. Díaz-Rubio) This work was supported in part by the European Commission through the H2020 ARIADNE project under grant 871464.

A. Díaz-Rubio, S. Kosulnikov, and S.A. Tretyakov are with the Department of Electronics and Nanoengineering, Aalto University, FI-00079 Aalto, Finland (e-mail: ana.diazrubio@aalto.fi; sergei.tretyakov@aalto.fi). neglecting dissipation loss) performance for arbitrary tilt angles, both for reflection, e.g [16]-[22] and refraction, e.g. [15], [23]-[27].

However, the theory and design of advanced metasurfaces for perfect anomalous reflection and refraction has been developed for infinite periodical arrays. Studies of the response of finite-sized panels have been based on various approximations and assumptions. Probably the simplest models of reflective metasurfaces consider each unit cell as a small mirror with a controllable reflection phase (sometimes tilted so that the reflection is in the desired direction). In this model, interactions of unit cells (meta-atoms) is completely ignored. In the vast majority of works on reconfigurable intelligent surfaces the classical reflectarray theory [10]-[12] is used. This theory is based on the notion of the local reflection coefficient, in the assumption that every "point" at the metasurface plane is characterized by a certain reflection coefficient. This is equivalent to the locally periodic approximation used in the theory and design of reflectarray antennas: the local reflection coefficient is calculated as a reflection coefficient of a regular infinite periodical array formed by identical cells, the same as the cell at the position where we want to know the reflection coefficient. This model takes into account interactions of unit cells assuming that the cell properties vary slowly on the wavelength scale.

Designs of theoretically perfect (no parasitic scattering) anomalous reflectors are based on the Floquet expansion of currents induced on periodical metasurfaces and on optimization of higher-order (evanescent) modes for suppression of scattering into all unwanted directions. Such metasurfaces cannot be characterized by a local reflection coefficient or the corresponding surface impedance. Recently, a more accurate model based on macroscopic reflection coefficients for each propagating Floquet harmonic of induced current has been introduced [28], and here we will use that approach, comparing the results with those based on the local reflection coefficient approximation.

Obviously, for any realistic application in telecommunications, it is necessary to account for finite sizes of metasurface panels and understand diffraction on edges and corners of the panels. For this reason, the main theoretical challenge to integrate RIS in wireless telecommunication systems is to find appropriate models for the metasurface response to external illuminations and calculating reflected and scattered fields (including both reflection and transmission) of finitesized metasurface mounted on walls with various reflection coefficients. Only some initial studies of these problems have been made so far, e.g. [29]-[34], see an overview in [35]. In the 
majority of works made by the communications community, the propagation channel is optimized by finding the optimal values of the local reflection coefficient (reflection phase at the position of each meta-atom). However, in reality, reflection from arrays is a collective phenomenon. These methods are applicable only under assumption that the metasurface is approximately uniform at the wavelength scale (in the theory of diffraction this model is called physical optics, e.g., [36]). For anomalously reflecting metasurfaces, this assumption holds only for small tilts of the reflected wave with respect to the specular-reflection angle. In addition to the need for an accurate model of the collective response of inhomogeneous metasurfaces, care should be taken to account for diffraction at the panel edges and corners.

Here, we develop an analytical model for metasurfaces mount at walls and used to control both reflection and transmission. In this work, we focus on metasurfaces for anomalous reflection, transmission, and beam splitting, where the metasurface structure is periodical along the tangential directions.

\section{PROBLEM FORMULATION}

In envisaged applications, metasurface panels can be illuminated from distant and low-directivity antennas (approximately, by plane waves at the reflector position) or by narrow beams of high-gain antennas (finite-size illuminated spots). Moreover, the size of the illuminated spot can be small or large as compared with the metasurface panel. Before discussing reflection and scattering from anomalous reflectors or windows, let us estimate the illuminated spot size for antennas with a given directivity.

It is usually enough to find an approximate estimation of the illuminated spot size, so it is possible to use simplifying assumptions. Let us assume that a wall with some mounted metasurface panels is illuminated with an antenna of directivity $D$, positioned at distance $r_{0}$ from the wall (see Fig. 1). Let us approximate the antenna radiation pattern by a cone (the angle $2 \theta$ ) with a constant intensity within the cone. The directivity of an antenna is inversely proportional to the solid angle $\Omega$, $D=4 \pi / \Omega$, through which the area of the illuminated spot can be found. The solid angle for a conical beam is $\Omega=$ $2 \pi(1-\cos \theta)=2 \pi\left(1-r_{0} / \sqrt{S / \pi+r_{0}^{2}}\right)$, where $S$ is the area

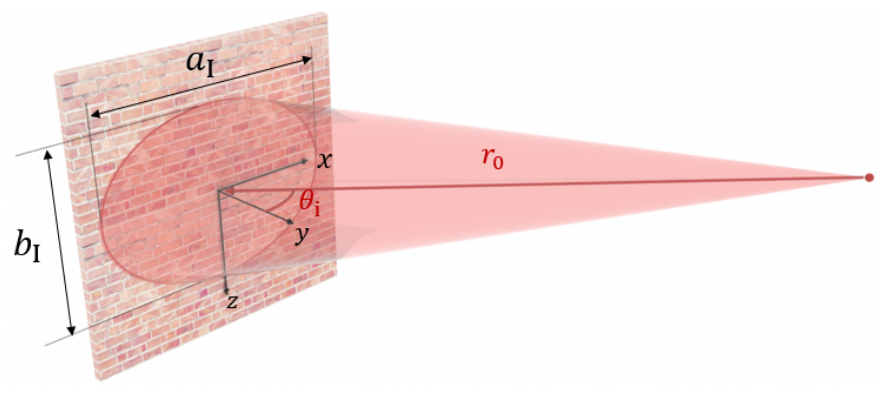

Fig. 1. Schematic representation of an illuminated area at a wall. The antenna is positioned at a distance $r_{0}$, and the incident angle is $\theta_{\mathrm{i}}$. of illumination. Now we can express $S$ in terms of $D$ and $r_{0}$. The result reads:

$$
S=\pi r_{0}^{2} \frac{4(D-1)}{(D-2)^{2}}=\pi r_{0}^{2}\left[\left(\frac{D}{D-2}\right)^{2}-1\right]
$$

For highly directive antennas, when $D \gg 1$, we have

$$
S \approx \pi r_{0}^{2} \frac{4}{D}
$$

In terms of antenna gain $G$ and efficiency $\varepsilon_{\mathrm{a}}$, substituting $D=$ $G / \varepsilon_{\mathrm{a}}$, we get

$$
S=\pi r_{0}^{2}\left[\left(\frac{G}{G-2 \varepsilon_{\mathrm{a}}}\right)^{2}-1\right]
$$

Here, for simplicity we have assumed normal illumination and a round shape of the illuminated spot. For oblique incidence, a more general expression for the axes of the illuminated ellipse $a_{\mathrm{I}}$ and $b_{\mathrm{I}}$ can be written in terms of the incidence angle $\theta_{\mathrm{i}}$ as follows:

$$
\begin{gathered}
a_{\mathrm{I}}=\frac{4 r_{0} \cos \theta_{\mathrm{i}} \sqrt{D-1}(D-2)}{D^{2} \cos ^{2} \theta_{\mathrm{i}}-4 D+4} \\
b_{\mathrm{I}}=\frac{\sqrt{D^{2} \sin ^{2} \sigma_{1}-4 D+4}}{(D-2)\left(4 D-4-D^{2} \cos ^{2} \theta_{\mathrm{i}}\right)} 2 r_{0} \sigma_{2} \cos \theta_{\mathrm{i}}
\end{gathered}
$$

where

$$
\begin{gathered}
\sigma_{1}=\operatorname{acos}\left(\frac{D-2}{D}\right)-\operatorname{asin}\left(\frac{2 \sqrt{D-1}(D-2)\left(4 D-4-D^{2} \cos ^{2} \theta_{\mathrm{i}}\right)}{D^{2} \cos \left(\theta_{i}+\operatorname{acos}\left(\frac{D-2}{D}\right)\right) \sigma_{2}}\right) \\
\sigma_{2}=\sqrt{16(D-1)^{2}-\left(8 D^{3}-D^{4}-8 D^{2}\right) \cos ^{2} \theta_{\mathrm{i}}}
\end{gathered}
$$

Finally, the resulting surface area of the illuminated ellipse can be simply calculated as a multiplication product of the axes $S_{\text {ellipse }}=\pi a_{\mathrm{I}} b_{\mathrm{I}} / 4$.

TABLE I

NUMERICAL EXAMPLE OF THE ILLUMINATED ELLIPSE AREA AS A FUNCTION OF THE INCIDENCE ANGLE $\theta_{\mathrm{i}}$, FOR $r_{0}=5 \mathrm{M}$ AND DIRECTIVTY $D=1000$

\begin{tabular}{|c|c|c|c|c|c|}
\hline$\theta_{\mathrm{i}}$, deg. & $b_{\mathrm{I}}, \mathrm{m}$ & $b_{\mathrm{I}} /\left.\lambda\right|_{40 \mathrm{GHz}}$ & $a_{\mathrm{I}}, \mathrm{m}$ & $a_{\mathrm{I}} /\left.\lambda\right|_{40 \mathrm{GHz}}$ & $S, \mathrm{~m}^{2}$ \\
\hline \hline 0 & 0.63 & 84.45 & 0.6334 & 84.45 & 0.3151 \\
\hline 20 & 0.63 & 84.46 & 0.6744 & 89.92 & 0.3356 \\
\hline 40 & 0.63 & 84.57 & 0.8292 & 110.56 & 0.4131 \\
\hline 60 & 0.64 & 84.97 & 1.2822 & 170.96 & 0.6418 \\
\hline 80 & 0.68 & 90.49 & 4.1880 & 558.40 & 2.2325 \\
\hline
\end{tabular}

Table I shows a numerical example of the values calculated using Eqs. (6) and (4) for a particular case of the beam illumination under different incidence angles $\theta_{\mathrm{i}}$ when $r_{0}=5 \mathrm{~m}$ and directivity $D=1000$. As expected, the surface area and the axis parameter $a_{\mathrm{I}}$ strongly depend on the incidence angle. For 5G millimeter-wave bands the size of the illumination area is usually large. Let us take as an example a frequency band around $40 \mathrm{GHz}$ and the relative ellipse dimensions calculated in Table I. We can see that the illumination area is larger than $84 \lambda \times 84 \lambda$ for all the incident angles. In this scenario, because of the large illumination area, it is necessary to design not only the electromagnetic properties but also the optimal area of the metasurface for the desired functionality. 
Depending on the size of the metasurface and the illuminating area we can distinguish two different scenarios. The first scenario realizes when the size of the illuminated spot is smaller than the metasurface and all the energy sent by the source impinges the metasurface. Here, the properties of the metasurface and the illumination profile will define the scattering properties. The second scenario is when the size of the illuminating spot is larger than the metasurface size, i.e., the source is partially illuminating the structure holding the metasurface. In this case, the properties of both metasurface and mounting structure have to be considered. In what follows, we will explore these scenarios for the case of metasurfaces mounted on a reflective wall. We will consider the scattering produced by both reflective and refractive metasurfaces.

\section{FAR-FIELD SCATTERING OF FINITE-SIZED REFLECTIVE METASURFACES MOUNTED ON A WALL}

In this section we study the far-field scattering of a reflective finite-sized metasurfaces mounted on a wall. To this end, we will develop a general theoretical model where the size of the metasurface, the size of the illumination area, and the illumination amplitude profile are arbitrarily defined. We will consider the scattering produced by a rectangular reflective metasurface with the size $a_{1} \times b_{1}$ (the surface of the metasurface is denoted by $\Omega_{1}$ ) located over an infinite and homogeneous partially reflective surface. In this analytical model, we will approximate the elliptical illumination area by a rectangular area $a_{2} \times b_{2}$ (denoted by $S$ ) and outside this area, the incident fields are assumed to be zero. The area $\Omega_{2}=S-\Omega_{1}$ is the illuminated area not filled by the metasurface, i.e, the area where the incoming waves impinge the homogeneous wall. A schematic representation of the considered geometry is shown in Fig. 2.

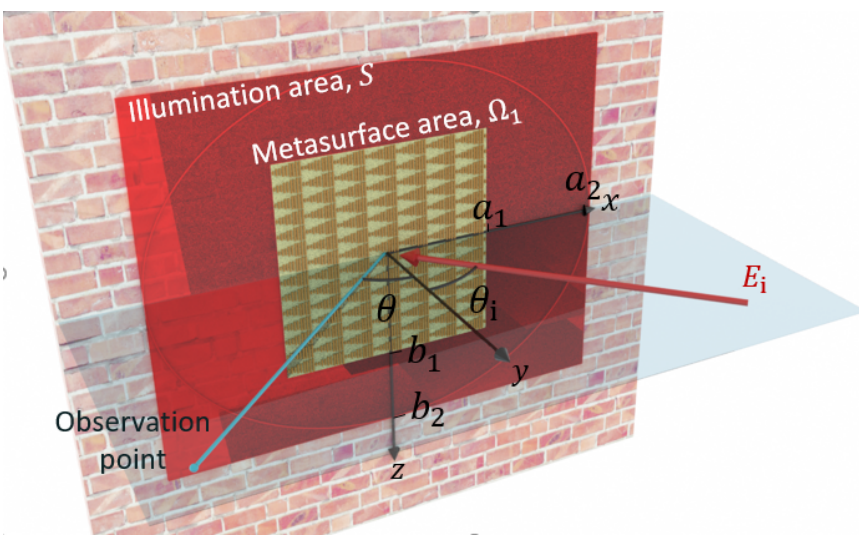

Fig. 2. Schematic representation of a reflective metasurface of the size $a_{1} \times b_{1}$ mounted over an impenetrable wall. The source (a directive antenna) illuminates the area $a_{2} \times b_{2}$.

We start the analysis by defining the total tangential field components in the illumination area as a combination of the incident and reflected fields. In order to calculate the fields reflected and scattered by the metasurface mounted at the wall, we define a Huygens volume that encloses the currents induced at the illuminated part of the wall and on the metasurface. As is well known, it is possible to introduce equivalent Huygens surface current densities based on either scattered or total fields at the Huygens surface (e.g., [36]). Here, we work with the total fields and introduce the equivalent Huygens surface currents as

$$
\mathbf{j}_{\mathrm{e}}^{+}=\hat{\mathbf{y}} \times\left.\mathbf{H}^{+}\right|_{y=0}, \quad \mathbf{j}_{\mathrm{m}}^{+}=-\hat{\mathbf{y}} \times\left.\mathbf{E}^{+}\right|_{y=0}
$$

where the superscript ' + ' marks the total fields and the surface currents on the illuminated side of the wall. Notice that the Huygens currents at the shadow side of the wall are zero, $\mathbf{j}_{\mathrm{e}}^{-}=\mathbf{j}_{\mathrm{m}}^{-}=0$, because fields do not penetrate the wall. The electric and magnetic fields on the illuminated side of the wall can be defined using the following expression

$$
\boldsymbol{\Upsilon}^{+}= \begin{cases}\boldsymbol{\Upsilon}_{\mathrm{i}}+\boldsymbol{\Upsilon}_{\mathrm{rm}} & \text { if }(x, z) \in \Omega_{1} \\ \boldsymbol{\Upsilon}_{\mathrm{i}}+\boldsymbol{\Upsilon}_{\mathrm{rw}} & \text { if }(x, z) \in \Omega_{2} \\ 0 & \text { otherwise }\end{cases}
$$

where the six-vector $\Upsilon=(\mathbf{E}, \mathbf{H})$ contains the components of the total electric and magnetic fields. Applying the superposition principle, the fields over the metasurface area can be written as a combination of the incident fields, $\boldsymbol{\Upsilon}_{i}$, and the fields reflected by the metasurface, $\boldsymbol{\Upsilon}_{\mathrm{rm}}$. In a similar manner, the field over the illuminated area where there is no metasurface is a combination of the incident field and the field reflected by the wall, denoted by $\Upsilon_{\mathrm{rw}}$.

\section{A. Uniform illumination}

We start from the case when the incident field inside the illuminated spot can be approximated by a plane wave. This corresponds to the step-function approximation of the radiation pattern of the illuminating antenna. Following the above definition, the incident fields $\mathbf{E}_{\mathrm{i}}$ and $\mathbf{H}_{\mathrm{i}}$ are present over the entire illuminated area and, in the case of TE-polarization (electric fields oriented in the $z$-direction), can be written as

$$
\begin{array}{r}
\mathbf{E}_{\mathrm{i}}=E_{0} e^{-j k\left(\sin \theta_{\mathrm{i}} x-\cos \theta_{\mathrm{i}} y\right)} \hat{\mathbf{z}} \\
\mathbf{H}_{\mathrm{i}}=-\frac{E_{0}}{\eta_{0}}\left(\cos \theta_{\mathrm{i}} \hat{\mathbf{x}}+\sin \theta_{\mathrm{i}} \hat{\mathbf{y}}\right) e^{-j k\left(\sin \theta_{\mathrm{i}} x-\cos \theta_{\mathrm{i}} y\right)}
\end{array}
$$

with $\theta_{\mathrm{i}}$ being the incidence angle of the illuminating wave, and $\eta_{0}=\sqrt{\mu_{0} / \varepsilon_{0}}$ is the wave impedance of the background medium. The reflected fields that correspond to the equivalent electric and magnetic surface currents in the region $\Omega_{2}$ are also plane waves (in the physical-optics approximation) and can be written as

$$
\begin{array}{r}
\mathbf{E}_{\mathrm{rw}}=E_{0} R e^{-j k\left(\sin \theta_{\mathrm{i}} x+\cos \theta_{\mathrm{i}} y\right)} \hat{\mathbf{z}} \\
\mathbf{H}_{\mathrm{rw}}=\frac{E_{0}}{\eta_{0}} R\left(\cos \theta_{\mathrm{i}} \hat{\mathbf{x}}-\sin \theta_{\mathrm{i}} \hat{\mathbf{y}}\right) e^{-j k\left(\sin \theta_{\mathrm{i}} x+\cos \theta_{\mathrm{i}} y\right)}
\end{array}
$$

where $R$ is the reflection coefficient of the homogeneous wall.

To model the reflected fields in the area of the metasurface, $\Omega_{1}$, we take into account that due to the periodicity of the metasurface, the scattered field can be expressed as a combination of Floquet harmonics. In our case, because we are interested in the values of the scattered fields in the far-field, only propagating harmonics will be considered (the contribution of edge-scattering due to evanescent harmonics is 
estimated in paper [28]). The reflected fields over the area of the metasurface read [28]

$$
\begin{gathered}
\mathbf{E}_{\mathrm{rm}}=E_{0} \sum_{n} r_{n} e^{-j k\left(\sin \theta_{\mathrm{r} n} x+\cos \theta_{\mathrm{r} n} y\right)} \hat{\mathbf{z}} \\
\mathbf{H}_{\mathrm{rm}}=\frac{E_{0}}{\eta_{0}} \sum_{n} r_{n}\left(\cos \theta_{\mathrm{r} n} \hat{\mathbf{x}}-\sin \theta_{\mathrm{r} n} \hat{\mathbf{y}}\right) e^{-j k\left(\sin \theta_{\mathrm{r} n} x+\cos \theta_{\mathrm{r} n} y\right)}
\end{gathered}
$$

where $r_{n}$ are the complex macroscopic reflection coefficients of the propagating Floquet harmonics, and $\theta_{\mathrm{r} n}$ define the directions of propagation of each reflected plane-wave harmonic. Note that the coefficients $r_{n}$ depend on the incidence angle. For a metasurface modeled by a given periodically varying surface impedance, these coefficients can be calculated using the mode-matching method [37], [38]. Importantly, these modal reflection coefficients are complex-valued constants, they do not depend on the spatial coordinates over the metasurface panel.

IIThe next step is to define the equivalent Huygens currents according to (7) and calculate the scattered fields created by these currents. On one side, this is the approach used in the physical optics, since we neglect field perturbations in the vicinity of the metasurface edges and propagation of edge-reflected surface waves along the reflector. On the other side, we go beyond the physical optics approximation using the macroscopic reflection coefficients, not assuming the local (or locally-periodical) approximation and taking into account collective nature of reflections from arrays and spatial dispersion of the metasurface structure. Let us also highlight that although direct scattering from edge inhomogeneities of evanescent modes of the metasurface is neglected, the presence of these modes is taken into account in calculation of the macroscopic reflection coefficients $r_{n}$.

In this work, we calculate the reflected and scattered fields in the far zone, using the far-field model that is valid under the following assumptions: $|\mathbf{r}| \gg \lambda,|\mathbf{r}| \gg \max \left(a_{2}, b_{2}\right)$, and $\max \left(a_{2}^{2} /|\mathbf{r}|, b_{2}^{2} /|\mathbf{r}|\right) \ll \lambda$, where $\mathbf{r}$ is the position vector from the center of the illuminated area to the observation point. That is, the distance to the observation point is large compared to the wavelength and to the size of both the metasurface plate and the illuminated area. Under these assumptions, the reflected field can be found as [28], [36]

$\mathbf{E}_{\mathrm{sc}}(\mathbf{r}) \approx \frac{j k}{4 \pi} \frac{e^{-j k|\mathbf{r}|}}{|\mathbf{r}|} \int_{S} e^{j k \hat{\mathbf{r}} \cdot \mathbf{r}^{\prime}} \hat{\mathbf{r}} \times\left[\eta_{0} \hat{\mathbf{r}} \times \mathbf{j}_{\mathrm{e}}\left(\mathbf{r}^{\prime}\right)+\mathbf{j}_{\mathrm{m}}\left(\mathbf{r}^{\prime}\right)\right] d x^{\prime} d z^{\prime}$

where $\hat{\mathbf{r}}$ is the unit vector along $\mathbf{r}$, pointing from the center of the metasurface plate to the observation point, and $\hat{\mathbf{r}}^{\prime}$ defines a certain point of the metasurface plane with coordinates $\left(x^{\prime}, 0, z^{\prime}\right)$. We note that the presented method can be used also for calculation of fields also in the near zone, by using the exact value of the Green function under the integral. The only limitation is that the distance from the metasurface plane to the observation point is assumed to be large as compared to the distance between meta-atoms of the metasurface.

Let us consider the scattered fields in the incidence plane $(\phi=\pi / 2)$. In this case, the reflected electric field has only $z$-component, and the expression of the scattered electric field can be simplified as

$$
E_{\mathrm{sc} z} \approx \frac{j k}{4 \pi} \frac{e^{-j k|\mathbf{r}|}}{|\mathbf{r}|} \int_{S} e^{j k \sin \theta x^{\prime}}\left[\eta_{0} H_{x}^{+}+\cos \theta E_{z}^{+}\right] d x^{\prime} d z^{\prime}
$$

where $\theta$ is the observation angle. In this expression the $H_{x}^{+}$and $E_{z}^{+}$represent the tangential components of the total magnetic and electric fields at the metasurface and at the illuminated part of the wall.

Calculating this integral for the total field defined in Eqs. (9)-(14), we obtain the following expression for the scattered electric field:

$$
\begin{aligned}
E_{\mathrm{sc} z}= & \frac{j k}{\pi} \frac{e^{-j k|\mathbf{r}|}}{|\mathbf{r}|} E_{0} \\
& {\left[a_{2} b_{2}\left((1+R) \cos \theta-(1-R) \cos \theta_{\mathrm{i}}\right) \operatorname{sinc}\left(k a_{\mathrm{ef}}\right)\right.} \\
& \left.+a_{1} b_{1} \sum_{n}\left(r_{n}-R \delta_{n}\right)\left(\cos \theta+\cos \theta_{\mathrm{r} n}\right) \operatorname{sinc}\left(k a_{\mathrm{ef} n}\right)\right]
\end{aligned}
$$

where $\delta_{n}$ is the Kronecker delta $\left(\delta_{0}=1\right.$ and $\left.\delta_{n \neq 0}=0\right)$. The parameters $a_{\mathrm{ef}}=\left(\sin \theta-\sin \theta_{\mathrm{i}}\right) a_{2}$, and $a_{\mathrm{ef} n}=(\sin \theta-$ $\left.\sin \theta_{\mathrm{r} n}\right) a_{1}$ represent the effective size of the metasurface for each reflected propagating Floquet mode.

For a clear representation, it is convenient to normalize the pattern so that the absolute value of its maximum for a PEC plate of the same size as the illuminated area is unity:

$$
\begin{aligned}
F_{\mathrm{sc} z}= & \frac{-1}{2 \cos \theta_{\mathrm{i}}}\left[\left((1+R) \cos \theta-(1-R) \cos \theta_{\mathrm{i}}\right) \operatorname{sinc}\left(k a_{\mathrm{ef}}\right)\right. \\
& \left.+\frac{a_{1} b_{1}}{a_{2} b_{2}} \sum_{n}\left(r_{n}-R \delta_{n}\right)\left(\cos \theta+\cos \theta_{\mathrm{r} n}\right) \operatorname{sinc}\left(k a_{\mathrm{ef} n}\right)\right]
\end{aligned}
$$

As a numerical example, we will analyse a metasurface that is mounted on a wall whose reflective coefficient is defined as $R=0.6$. The considered example metasurface is designed for reflecting plane waves coming from an illumination angle $\theta_{\mathrm{id}}=70^{\circ}$ into the reflection angle $\theta_{\mathrm{rd}}=0^{\circ}$. For these design conditions, the period of the metasurface reads $D=\lambda /\left|\sin \theta_{\text {id }}-\sin \theta_{\text {rd }}\right|$ [16]. Following the Floquet theory, the periodicity of the metasurface defines the propagation directions of diffracted modes which depend on the illumination angle, the period, and the frequency. In this particular example, three different Floquet harmonics can propagate. The harmonic $n=0$ corresponds to specular reflection, $\theta_{\mathrm{r} 0}=70^{\circ}$. The desired anomalous reflection is offered by the harmonic $n=-1$ that propagates in the normal direction, $\theta_{\mathrm{r}-1}=0^{\circ}$. Finally, the existence of propagating harmonic $n=-2$ tells that waves can be reflected also to the opposite direction of the incident wave (retroreflection response), at $\theta_{\mathrm{r}-2}=-70^{\circ}$.

As it has been extensively studied in the literature [13][17], the distribution of reflected power among the different propagating modes depends on the metasurface design. Let us start by considering a metasurface that performs perfect anomalous reflection, i.e, all the power impinging at $\theta_{\mathrm{i}}=70^{\circ}$ is sent into the normal direction. In this particular example, to warranty that all the energy is reflected into the desired 


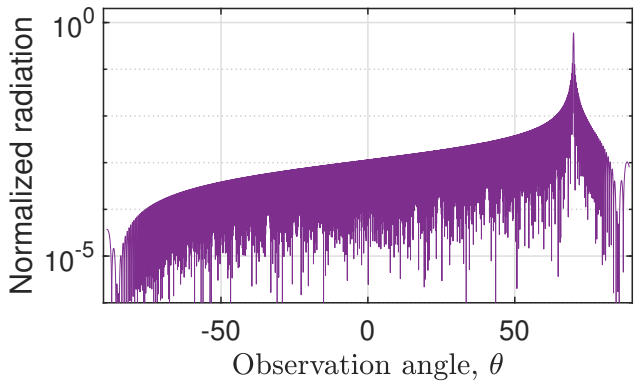

(a)

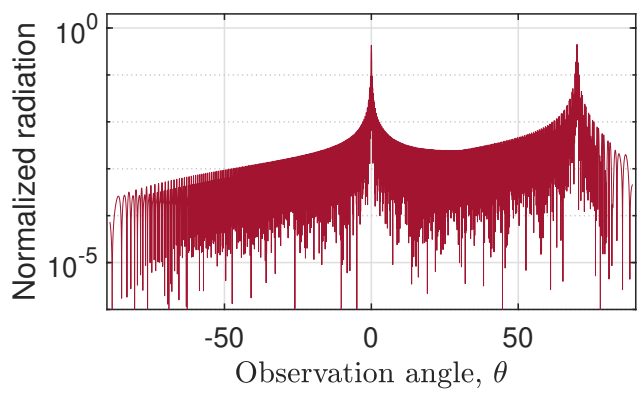

(b)

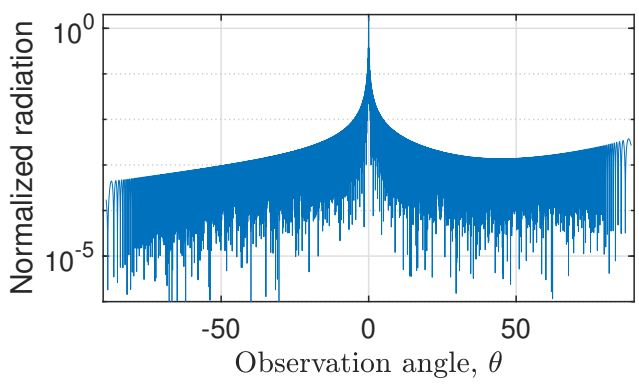

(c)

Fig. 3. Numerical study of the far-field scattering produced by a metasurface designed to produce perfect anomalous reflection for an illumination angle $\theta_{\mathrm{i}}=70^{\circ}$ and a reflection angle $\theta_{\mathrm{r}}=0^{\circ}$ for different sizes of the metasurface, when the illumination area is $100 \lambda \times 100 \lambda$. (a) Scattered field from the wall without the metasurface. (b) The size of the metasurface $50 \lambda \times 50 \lambda$. (c) The size of the metasurface $100 \lambda \times 100 \lambda$.

direction the amplitude of the Floquet harmonic $n=-1$ must be $r_{-1}=\sqrt{\left(\cos \theta_{\text {id }} / \cos \theta_{\text {rd }}\right)} \approx 0.59$ [15].

Figure 3 presents the far-field scattering pattern for different sizes of the metasurface when the illuminated area is $100 \lambda \times 100 \lambda$. First, Fig. 3(a) shows the far-field scattering pattern without the metasurface, i.e., all the illuminating wave impinges the uniform wall. As expected, the scattering pattern has a single maximum in the specular direction. The maximum amplitude is smaller than one because of the losses in the wall. Next, let us consider an intermediate scenario where the metasurface partially covers the illumination spot. In this case, we will take an example when the size of the metasurface is $50 \lambda \times 50 \lambda$. Figure $3(\mathrm{~b})$ shows the radiation pattern where we can see two directions of maximum reflection that correspond to both anomalous and specular reflections. Finally, we analyze the reflection from a metasurface that completely covers the illumination area. We note that this case is equivalent to illumination of a large metasurface with a focused beam that illuminates only a part of the metasurface. This surface creates a reflected beam with a single maximum of radiation in the anomalous direction. It is important to notice that controlling the relative size of the illumination area and the metasurface we can easily implement a beam splitter and control the amount of power sent into each of these two directions.

Notice that the assumption of a plane-wave field illuminating a finite-size area of the wall implies discontinuities of the fields at the edges of the illumination area, which are in reality not present. As it was numerically demonstrated in [28], the discontinuity at the edge of the metasurface is a good approximation for large metasurfaces and the effect of the evanescent fields at the borders can be neglected. However, this assumption may lead to inaccuracies in the estimations of scattered fields in side lobes, defined by diffraction at the edges of the illuminated spot. The effects of the discontinuity in the edge of the illumination area will be studied in next subsection.

The above relations for the fields in the far zone are applicable to the case when the distance to the observation point is large compared to both the metasurface size and to the size of the illuminated area. In practice, when the illuminating antenna is weakly directive, it can happen that the illuminated area is so wide that the observation point is in the near zone with respect to the size of the illuminated area. In this case, a reasonable approximation is to assume that the field reflected from the wall in the absence of metasurface is a plane wave, and not a spherical wave ad in the above model.

$$
\begin{gathered}
E_{\mathrm{sc} z}=\frac{j k}{\pi} \frac{e^{-j k|\mathbf{r}|}}{|\mathbf{r}|} E_{0} a_{1} b_{1} \sum_{n}\left(r_{n}-R \delta_{n}\right)\left(\cos \theta+\cos \theta_{\mathrm{r} n}\right) \operatorname{sinc}\left(k a_{\mathrm{ef} n}\right) \\
+R E_{0} e^{-j k\left(\sin \theta_{\mathrm{i}} x+\cos \theta_{\mathrm{i}} y\right)}
\end{gathered}
$$

Here, the last term is the plane wave reflected from the infinite uniformly illuminated wall. Diffraction due to the inhomogeneity of the currents flowing on the wall at the metasurface edges is taken into account by the terms in the expression for the spherical wave that are proportional to $R$. Expression (19) is applicable when the distance to the observation point is large compared to the size of the metasurface but small compared to the size of the illuminated area.

\section{B. Non-uniform illumination}

To create a model for realistic beam illuminations, where the amplitude of the incident field smoothly decays from the center of the illumination area to its edges, we will model the amplitude of the incident wave by a cosine function. We select this particular model because it allows us to derive fully analytical expressions for the scattering patterns. In addition, because the variation of the incident field amplitude in the transverse plane is slow at the wavelength scale, we assume that both the incident and reflected fields can be locally considered as plane waves with slowly varying amplitudes. For 


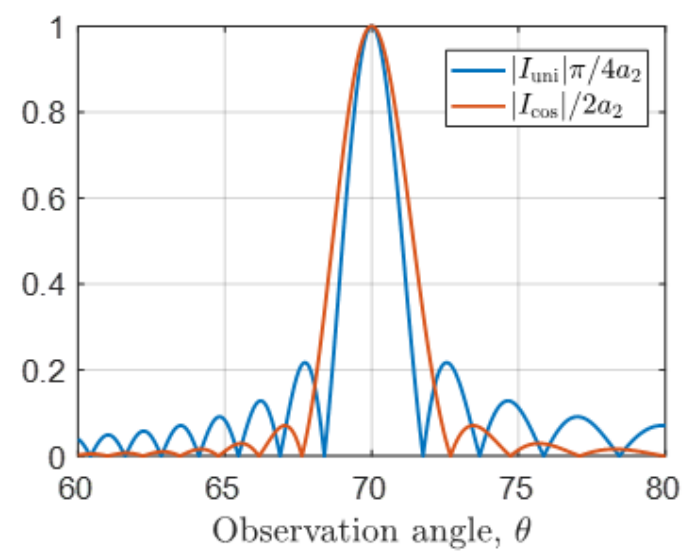

Fig. 4. Effect of the illumination amplitude profile on the scattering pattern. Comparison between the normalized radiation patterns at the plane $\phi=\pi / 2$ associated with the incident field for the uniform and cosine profiles. The size of the illumination area is defined by $a_{2}=50 \lambda$.

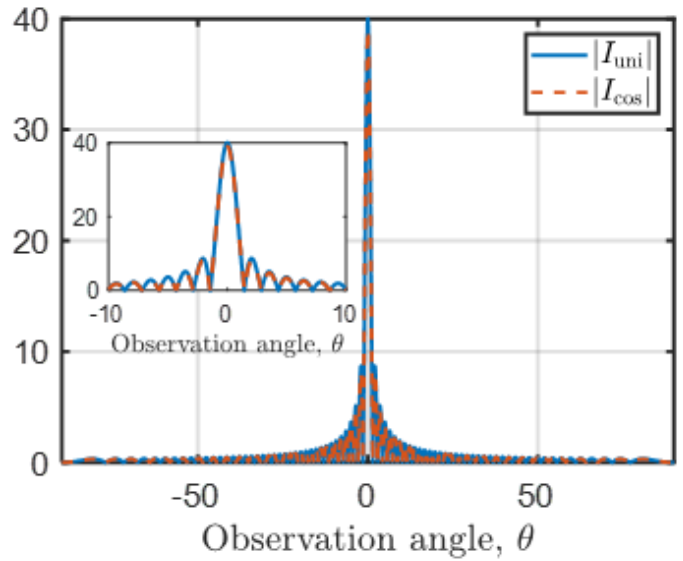

Fig. 5. Comparison between the radiation patterns associated with the metasurface radiation for uniform and non-uniform illuminations (cosine profile) when $\theta_{\mathrm{r} n}=0^{\circ}, a_{2}=50 \lambda$, and $a_{1}=20 \lambda$.

example, the tangential component of the illuminating fields at $y=0$ we write as

$$
\begin{array}{r}
E_{\mathrm{iz}}(x, z)=E(x, z) e^{-j k\left(\sin \theta_{\mathrm{i}} x-\cos \theta_{\mathrm{i}} y\right)} \\
H_{\mathrm{ix}}(x, z)=-\frac{E(x, z)}{\eta_{0}} \cos \theta_{\mathrm{i}} e^{-j k\left(\sin \theta_{\mathrm{i}} x-\cos \theta_{\mathrm{i}} y\right)}
\end{array}
$$

where $E(x, z)$ represents a slow varying function that defines the illumination profile. The same assumption is made for the reflected fields on the wall and the metasurface.

Using this plane-wave approximation and restricting the study to the scattered fields in the plane $\phi=\pi / 2$, we will define the illumination profile as $E(x)=\cos \left(\frac{\pi}{2 a_{2}} x\right)$. First, we will consider the effect of the non-uniform illumination profile on the term associated with the incident field [the first term in Eq. (18)]. In this case, the effect of the non-uniform illumination appears in an integral of the form

$$
I_{\cos }(\theta)=\int_{-a_{2}}^{a_{2}} \cos \left(\frac{\pi}{2 a_{2}} x\right) e^{j \Phi_{\mathrm{i}}(\theta) x} d x=4 a_{2} \pi \frac{\cos \left(\Phi_{\mathrm{i}} a_{2}\right)}{\pi^{2}-4 \Phi_{\mathrm{i}}^{2} a_{2}^{2}}
$$

where $\Phi_{\mathrm{i}}(\theta)=k\left(\sin \theta-\sin \theta_{\mathrm{i}}\right)$ contains the phase information of the incident field. For comparison, let us write the same integral for the uniform illumination case:

$$
I_{\text {uni }}(\theta)=\int_{-a_{2}}^{a_{2}} e^{j \Phi(\theta) x} d x=2 a_{2} \frac{\sin \left(\Phi a_{2}\right)}{\Phi a_{2}}
$$

The same expressions are also applicable to the field reflected by the wall. Both functions have the maximum at $\theta=\theta_{\mathrm{i}}$. However, the amplitude of the radiation pattern at this observation angle is not the same, $\mid I_{\cos }\left(\theta_{\mathrm{i}} / I_{\text {uni }}\left(\theta_{\mathrm{i}}\right) \mid=2 / \pi\right.$. This ratio represents the reduction of the illuminating power due to the cosine profile. From these results we can expect that the amount of specularly reflected power will be reduced.

For a better comparison between the two radiation patterns, Figure 4 shows the normalized reflection patterns when $\theta_{\mathrm{i}}=$ $70^{\circ}$ and $a_{2}=50 \lambda$. From this comparison we can see that, as expected, the non-uniformity of illumination and a smooth tapering of the field at the edges of the illuminated spot reduce the amplitude of the side lobes. In addition, the width of the main lobe increases with the cosine profile.

Now, let us consider the second term in in Eq. (18), that models the scattering produced by the metasurface. In the general case, this term is proportional to the following integral:

$$
\begin{gathered}
I_{\cos }(\theta)=\int_{-a_{1}}^{a_{1}} \cos \left(\frac{\pi}{2 a_{2}} x\right) e^{j \Phi_{\mathrm{r} n}(\theta) x} d x= \\
=4 a_{2} \frac{\pi \sin \left(\frac{a_{1} \pi}{2 a_{2}}\right) \cos \left(\Phi_{\mathrm{r} n} a_{1}\right)-2 a_{2} \Phi_{\mathrm{r} n} \cos \left(\frac{a_{1} \pi}{2 a_{2}}\right) \sin \left(\Phi_{\mathrm{r} n} a_{1}\right)}{\pi^{2}-4 \Phi_{\mathrm{r} n}^{2} a_{2}^{2}}
\end{gathered}
$$

with $\Phi_{\mathrm{r} n}=k\left(\sin \theta-\sin \theta_{\mathrm{r} n}\right)$ being the phase profile of each Floquet harmonic. Using this expression, we can study different regimes depending on the metasurface size. First, let us consider a metasurface that is smaller than the illumination area $a_{1}<a_{2}$ while both $a_{1}$ and $a_{2}$ are much larger than the wavelength. In this case, metasurface is only illuminated by the central part of the beam, and the variation of the illuminated field over the metasurface is small. As an example, we compare the normalized radiation pattern associated with the metasurface when $a_{2}=50 \lambda$ and $a_{1}=20 \lambda$, for $\theta_{\mathrm{r} n}=0^{\circ}$. Figure 5 shows the comparison for different observation angles. We can see that for this configuration there is no appreciable difference between the two radiation patterns associated with the metasurface. This means that, in this particular example, the amplitude of the radiation lobe in the specular direction will be reduced due to reduction of the illumination power, while the radiation lobe at the anomalous reflection will remain the same as for the uniform illumination.

The second regime corresponds to metasurfaces with a large area that completely fill the illumination area: $a_{1}=a_{2}$. In this case, Eq. (24) becomes identical to Eq. (22), and the radiation pattern from the metasurface will be affected by the cosine profile in the same way as the pattern associated with the incident field and the reflections from the wall. Finally, it is interesting to mention the regime where a metasurface with a small area completely covers the illumination area. In this case, the amplitude of the incident field considerably changes between different periods of the metasurface and amplitudes 
of the Floquet harmonies should be calculated taking into consideration this variation, i.e., without the assumption of locally plane-wave illumination.

\section{Comparison of performance of phase-gradient metasur-} faces and perfect anomalous reflectors

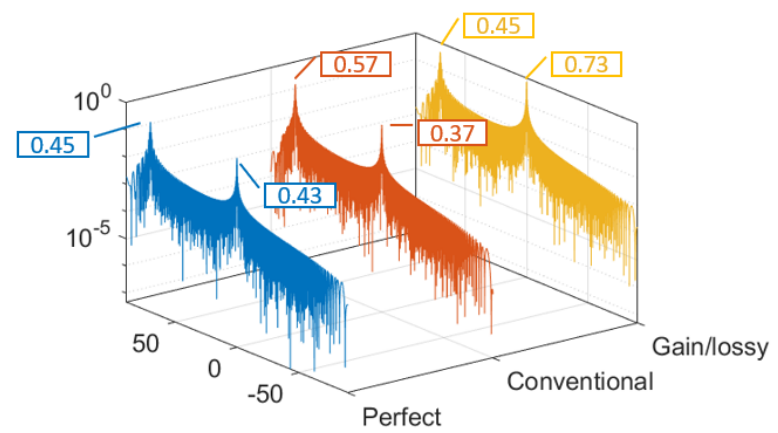

(a)

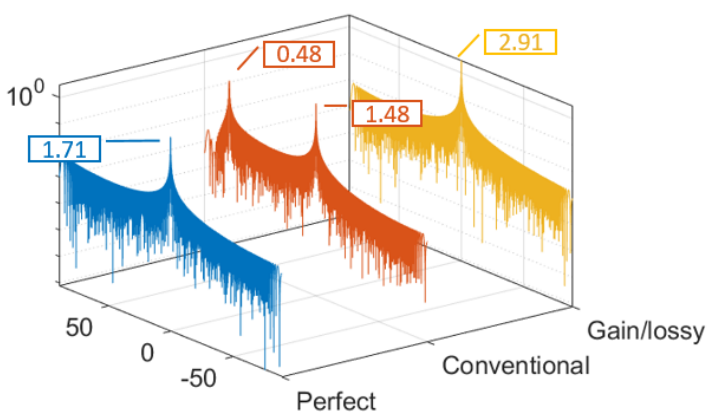

(b)

Fig. 6. Scattering patterns for different types of anomalous reflectors for the illumination angle $\theta_{\mathrm{i}}=70^{\circ}$ and the reflection angle $\theta_{\mathrm{r}}=0^{\circ}$. (a) The illumination area is $100 \lambda \times 100 \lambda$ and the metasurface size is $50 \lambda \times 50 \lambda$. (b) The metasurface completely covers the illuminated area $100 \lambda \times 100 \lambda$.

In the above examples, we considered a theoretically perfect design of anomalous reflectors, where scattering into all unwanted Floquet harmonics is suppressed by proper optimization of higher-order, surface-bound modes [16], [17], [19], [21]. For infinite structures, this type of anomalous reflectors warranties that all the incident power is reflected into the desired direction or directions. In the same manner, finite-size metasurfaces designed as perfect anomalous reflectors maximize the amplitude of the radiation pattern in the desired direction. However, there are other types of anomalous reflectors with different scattering properties that

TABLE II

SCATTERING PROPERTIES OF INFINITE ANOMALOUS REFLECTORS BASED ON DIFFERENT DESIGN APPROACHES (DESIGN TARGET: $\theta_{\mathrm{i}}=70^{\circ}$ AND

$$
\left.\theta_{\mathrm{r}}=0^{\circ}\right) \text {. }
$$

\begin{tabular}{|l|c|c|c|c|}
\hline & $\begin{array}{c}r_{0} \\
\left(70^{\circ}\right)\end{array}$ & $\begin{array}{c}r_{-1} \\
\left(0^{\circ}\right)\end{array}$ & $\begin{array}{c}r_{-2} \\
\left(-70^{\circ}\right)\end{array}$ & $P_{\text {out }} / P_{\text {in }}$ \\
\hline Perfect anomalous reflectors & 0.00 & 0.59 & 0.00 & 1.01 \\
\hline Linear phase gradient & 0.48 & 0.51 & 0 & 0.99 \\
\hline Active/lossy reflectors & 0.00 & 1.00 & 0.00 & 2.94 \\
\hline
\end{tabular}

will produce different radiation patterns. In this subsection, we briefly explain the most common scenarios.

The most established and commonly used approach for the design of anomalous reflectors is based on realizations of a linear phase gradient of the local reflection coefficient (as in reflectarray antennas). As is well known, conventional inhomogeneous metasurfaces, defined by the local reflection coefficient with controllable reflection phase, inevitably produce parasitic reflections into all directions corresponding to all propagating Floquet modes [13]-[17]. Here, we present examples of numerical comparison of performance of phasegradient reflectors and theoretically perfect anomalous reflectors mounted on walls. We consider the same examples of metasurfaces designed to reflect waves incident from $\theta_{\mathrm{i}}=70^{\circ}$ to the normal direction, $\theta_{\mathrm{r}}=0^{\circ}$. Table II summarizes the calculated amplitudes of three propagating harmonics extracted from the analysis of the infinite structure, where we can see what part of the incident power is reflected in the specular direction. In this case, this wave interferes with the wave reflected by the wall, enhancing the radiation lobe at the specular direction. On the contrary, the radiation lobe in the normal direction is reduced. Figure 6 compares the radiation patterns for the different design strategies.

Let us stress the importance to properly calculate the amplitudes of the propagating harmonics and the total power scattered by the metasurface. If we consider an infinite metasurface performing anomalous reflection and assume the amplitude of the macroscopic reflection coefficient to be unity: $r_{-1}=1$, the power scattered by the metasurface will be defined $P_{\text {out }} / P_{\text {in }}=$ $\sum r_{n}^{2} \cos \theta_{\mathrm{r} n} / \cos \theta_{\mathrm{i}}$. For the same example of an anomalous reflector for $\theta_{\mathrm{i}}=0^{\circ}$ and $\theta_{\mathrm{r}}=70^{\circ}$, the power scattered is $P_{\text {out }} / P_{\text {in }} \approx 2.94$, meaning that the metasurface introduces energy to the system and microscopically there should be active elements to provide gain. In the tables and plots, this model is marked as "active/lossy", because for other incidence and reflection angles the corresponding reflector can be also lossy.

The effect of the design approach in the scattering properties becomes more significant when the size of the metasurface increases. For the same example, if the of metasurface completely covers the illuminated area $(100 \lambda \times 100 \lambda)$, perfect anomalous reflectors will send the incident power into a single beam in the desired direction. However, anomalous reflectors based on a linear phase gradient of reflection coefficient exhibit significant scattering into different parasitic harmonics, as is evident from Fig. 6(b).

The distribution of energy between the parasitic harmonics is also closely related with the design conditions. For example, if we change the design conditions of the anomalous reflector to $\theta_{\mathrm{i}}=0^{\circ}$ and $\theta_{\mathrm{r}}=70^{\circ}$, the number of propagating Floquet harmonics and the directions of their propagation are the same, however, the power distribution changes. Table III summarizes the scattering properties of infinite metamirrors designed for these operations. One can see that in the case of anomalous reflectors designed with a linear phase gradient the power is distributed among three Floquet harmonics. For this reason, the radiation pattern of these finite-size metamirrors contains three radiation lobes, as is shown in Fig. 7. It also important 
TABLE III

SCATTERING PROPERTIES OF INFINITE ANOMALOUS REFLECTORS BASED ON DIFFERENT DESIGN APPROACHES (DESIGN TARGET: $\theta_{\mathrm{i}}=0^{\circ}$ AND $\left.\theta_{\mathrm{r}}=70^{\circ}\right)$.

\begin{tabular}{|l|c|c|c|c|}
\hline & $\begin{array}{c}r_{1} \\
\left(70^{\circ}\right)\end{array}$ & $\begin{array}{c}r_{0} \\
\left(0^{\circ}\right)\end{array}$ & $\begin{array}{c}r_{-1} \\
\left(-70^{\circ}\right)\end{array}$ & $P_{\text {out }} / P_{\text {in }}$ \\
\hline Perfect anomalous reflectors & 1.71 & 0.00 & 0.00 & 1.00 \\
\hline Linear phase gradient & 1.50 & 0.24 & 0.73 & 1.00 \\
\hline Active/lossy reflectors & 1.00 & 0.00 & 0.00 & 0.34 \\
\hline
\end{tabular}

to note that a metamirror designed to produce anomalous reflection with a macroscopic reflection coefficient $r_{1}=1$ in this configuration will be overall lossy, and only $34 \%$ of the incident power will be reflected.

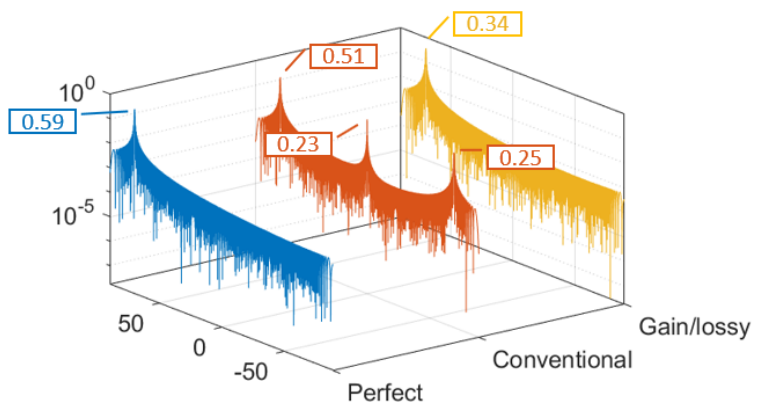

Fig. 7. Scattering patterns for different types of anomalous reflectors for the illumination angle $\theta_{\mathrm{i}}=0^{\circ}$ and the reflection angle $\theta_{\mathrm{r}}=70^{\circ}$ when the metasurface completely covers the illuminated area of the size $100 \lambda \times 100 \lambda$.

\section{FAR-FIELD SCATTERING OF A REFRACTIVE FINITE-SIZE METASURFACES MOUNTED ON A WALL}

The second example that we study is a refractive metasurface mounted over a reflective or/and absorbing wall. Here, the metasurface allows and controls wave transmission through the window region $\Omega_{1}$. However, the wall is still impenetrable, and fields do not penetrate though the wall outside of the

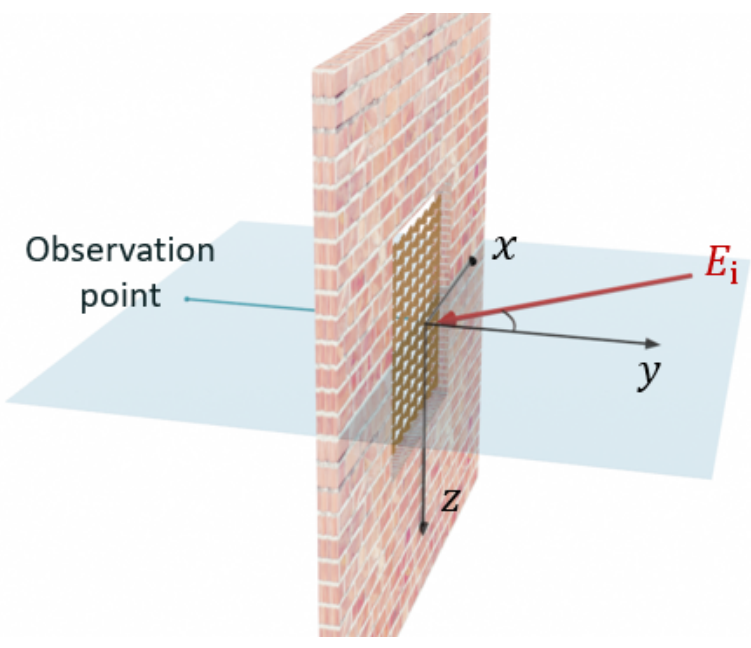

Fig. 8. Schematic representation of a refractive metasurface mounted in a window in an impenetrable wall. The metasurface controls the direction of beam transmitted through the window. metasurface area. Figure 8 shows a schematic representation of the problem. Using the same notations as in the previous example, the equivalent Huygens electric and magnetic surface current densities at the illuminated side of the wall are defined in terms of the total fields at the two sized of the metasurface and the wall as $\mathbf{j}_{\mathrm{e}}^{+}=\hat{\mathbf{y}} \times\left.\mathbf{H}^{+}\right|_{y=0}$ and $\mathbf{j}_{\mathrm{m}}^{+}=-\hat{\mathbf{y}} \times\left.\mathbf{E}^{+}\right|_{y=0}$ and the currents at the shadow side as $\mathbf{j}_{\mathrm{e}}^{-}=-\hat{\mathbf{y}} \times\left.\mathbf{H}^{-}\right|_{y=0}$ and $\mathbf{j}_{\mathrm{m}}^{+}=\hat{\mathbf{y}} \times\left.\mathbf{E}^{-}\right|_{y=0}$. The field in the illuminated side are defined using Eq. (8). However, the fields at the shadow side of wall are defined by

$$
\mathbf{\Upsilon}^{-}= \begin{cases}\Upsilon_{\mathrm{tm}} & \text { if }(x, z) \in \Omega_{1} \\ 0 & \text { otherwise }\end{cases}
$$

As in the previous example, the six-vectors $\Upsilon=(\mathbf{E}, \mathbf{H})$ contain both electric and magnetic fields.

The transmitted fields over the area of the metasurface, $\Omega_{1}$, read

$$
\begin{gathered}
\mathbf{E}_{\mathrm{tm}}=E_{0} \sum_{n} t_{n} e^{-j k\left(\sin \theta_{\mathrm{t} n} x-\cos \theta_{\mathrm{t} n} y\right)} \hat{\mathbf{z}} \\
\mathbf{H}_{\mathrm{tm}}=-\frac{E_{0}}{\eta_{0}} \sum_{n} t_{n}\left(\cos \theta_{\mathrm{t} n} \hat{\mathbf{x}}+\sin \theta_{\mathrm{t} n} \hat{\mathbf{y}}\right) e^{-j k\left(\sin \theta_{\mathrm{t} n} x+\cos \theta_{\mathrm{t} n} y\right)}
\end{gathered}
$$

where $t_{n}$ are the transmission coefficients of the propagating Floquet harmonics and $\theta_{\mathrm{t} n}$ defines the direction of propagation of each harmonic.

Now, we just need to integrate the equivalent Huygens electric and magnetic surface currents multiplied by the Green function. The integration over the illuminated area is the same as for reflectors, but the integration of the currents behind the wall produces an additional term in the expression of the scattered fields:

$$
\begin{aligned}
E_{\mathrm{sc} z} & \approx \frac{j k}{4 \pi} \frac{e^{-j k|\mathbf{r}|}}{|\mathbf{r}|}\left[\int_{S} e^{j k \sin \theta x^{\prime}}\left[\eta_{0} H_{x}^{+}\left(x^{\prime}\right)+\cos \theta E_{z}^{+}\left(x^{\prime}\right)\right] d x^{\prime} d z^{\prime}\right. \\
& \left.+\int_{S} e^{j k \sin \theta x^{\prime}}\left[\eta_{0} H_{x}^{-}\left(x^{\prime}\right)+\cos \theta E_{z}^{-}\left(x^{\prime}\right)\right] d x^{\prime} d z^{\prime}\right]
\end{aligned}
$$

Calculating the integrals, the final expression of the scattered field in the incidence plane reads

$$
\begin{aligned}
E_{\mathrm{sc} z}= & \frac{j k}{4 \pi} \frac{e^{-j k|\mathbf{r}|}}{|\mathbf{r}|} E_{0}\left[a_{2} b_{2}\left((1+R) \cos \theta-(1-R) \cos \theta_{\mathrm{i}}\right) \operatorname{sinc}\left(k a_{\mathrm{ef}}\right)\right. \\
+ & a_{1} b_{1} \sum_{n}\left(r_{n}-R \delta_{n}\right)\left(\cos \theta+\cos \theta_{\mathrm{r} n}\right) \operatorname{sinc}\left(k a_{\mathrm{ef} n}\right) \\
& \left.+a_{1} b_{1} \sum_{n} t_{n}\left(\cos \theta-\cos \theta_{\mathrm{t} n}\right) \operatorname{sinc}\left(k a_{\mathrm{ef} n}\right)\right] \\
E_{\mathrm{sc} z}= & \frac{j k}{4 \pi} \frac{e^{-j k|\mathbf{r}|}}{|\mathbf{r}|} E_{0}\left[a_{2} b_{2} R(\cos \theta-1) \cos \theta_{\mathrm{i}}\right) \operatorname{sinc}\left(k a_{\mathrm{ef}}\right) \\
+ & a_{1} b_{1} \sum_{n}\left(r_{n}-R \delta_{n}\right)\left(\cos \theta+\cos \theta_{\mathrm{r} n}\right) \operatorname{sinc}\left(k a_{\mathrm{ef} n}\right) \\
& \left.+a_{1} b_{1} \sum_{n} t_{n}\left(\cos \theta-\cos \theta_{\mathrm{t} n}\right) \operatorname{sinc}\left(k a_{\mathrm{ef} n}\right)\right]
\end{aligned}
$$


Following the same approach as in the study of reflective metasurfaces, we normalize the pattern so that the absolute value of its maximum for a PEC plate of the same size as the illuminated area is unity, and obtain

$$
\begin{gathered}
F_{\mathrm{sc} z}=\frac{-1}{2 \cos \theta_{\mathrm{i}}}\left[\left((1+R) \cos \theta-(1-R) \cos \theta_{\mathrm{i}}\right) \operatorname{sinc}\left(k a_{\mathrm{ef}}\right)\right. \\
+\frac{a_{1} b_{1}}{a_{2} b_{2}} \sum_{n}\left(r_{n}-R \delta_{n}\right)\left(\cos \theta+\cos \theta_{\mathrm{r} n}\right) \operatorname{sinc}\left(k a_{\mathrm{ef} n}\right) \\
\left.+\frac{a_{1} b_{2}}{a_{2} b_{2}} \sum_{n} t_{n}\left(\cos \theta-\cos \theta_{\mathrm{t} n}\right) \operatorname{sinc}\left(k a_{\mathrm{ef} n}\right)\right]
\end{gathered}
$$

As an example, we study a refractive metasurface with the area $10 \lambda \times 10 \lambda$ designed to produce anomalous refraction for an illumination angle $\theta_{\mathrm{i}}=70^{\circ}$ and a transmission angle $\theta_{\mathrm{t}}=0^{\circ}$, when the illuminated area is $20 \lambda \times 20 \lambda$. For simplicity we will also assume that the metasurface is designed to achieve perfect anomalous transmission, using methods described in [15], [23]-[26]. The scattering pattern for this scenario calculated using Eq. (31) is shown in Fig. 9. The scattering pattern has three radiation lobes at three different directions. First, on the illuminated side of the metasurface there is a radiation lobe corresponding to specular reflection from the impenetrable wall. The amplitude of this lobe depends on the area of the window covered by the metasurface. i.e., the larger area of the metasurface, the smaller amount of power is reflected into the specular direction. On the shadow side of the wall, we distinguish two radiation lobes: a radiation lobe in the normal direction that corresponds to the anomalous transmission, and a scattering lobe in the direction of the incident wave. It is important to stress that the role of the lobe in the direction of the incident wave is to cancel the incident field in the shadow. In this example of an impenetrable wall and a perfect anomalous refractor in the window, the total field behind the wall does not contain any beam in the direction of the incident wave, as this shadow scattering beam exactly cancels out the incident field behind the wall.

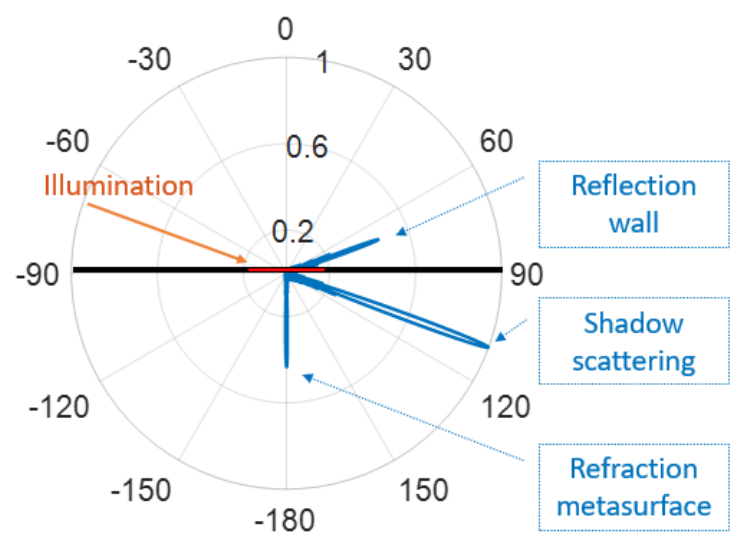

Fig. 9. Field patterns for a refractive metasurface with the area $10 \lambda \times 10 \lambda$ designed to produce anomalous refraction for the illumination angle $\theta_{\mathrm{i}}=70^{\circ}$ and the transmission angle $\theta_{\mathrm{t}}=0^{\circ}$ when the illuminated area is $20 \lambda \times 20 \lambda$. The partially reflective wall is modeled by $R=0.6$.
However, secondary lobes of the shadow scattering can affect the scattering pattern on the illuminated side, in front of the wall. In the plot of Fig. 9, the reflected field is dominated by specular reflection from the wall, and the weaker scattering due to inhomogeneities is not visible. To analyze these effects, we consider the same scenario as before, with the only difference that the wall is modeled as a perfect absorber, characterized by $R=0$ (see Fig. 10). In this case, the reflected and scattered field on the illuminated side does not contain a specularly reflected component, but there is scattered field due to both scattering at the metasurface edges and at the boundaries of the illuminated area (the shadow scattering term). The same figure shows the total field behind the metasurface, where we can see that the incident plane wave is completely suppressed, and only the radiation lobe associated to the anomalous refraction remains. The effect of shadow-current scattering at the edges of the illuminated area depends on the metasurface area and the angle of incidence $\theta_{\mathrm{i}}$, and it is present even in the limit case when the metasurface window area tends to zero.

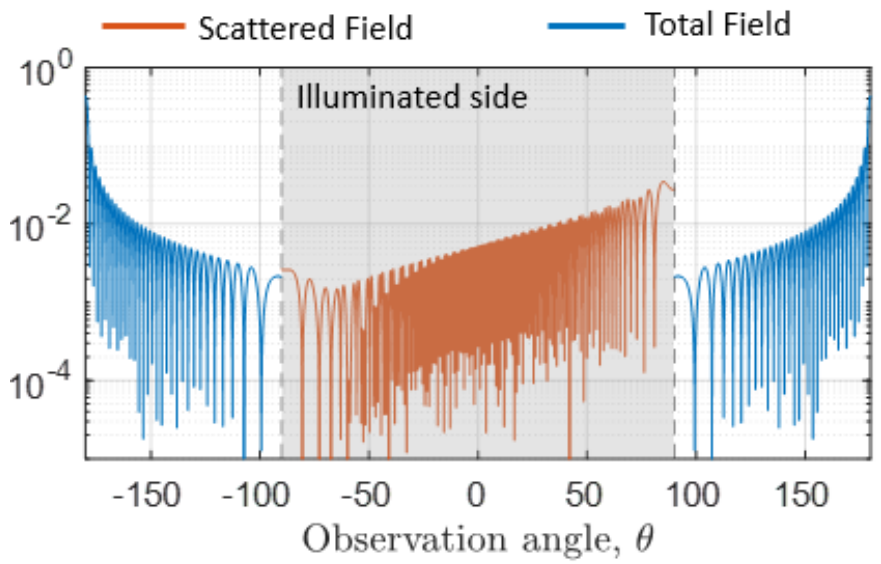

Fig. 10. Scattering patterns for a refractive metasurface with the area $10 \lambda \times$ $10 \lambda$ designed to produce anomalous refraction for the illumination angle $\theta_{\mathrm{i}}=$ $70^{\circ}$ and the transmission angle $\theta_{\mathrm{t}}=0^{\circ}$ when the illuminated area is $20 \lambda \times$ $20 \lambda$. The wall is modeled by $R=0$, acting as a perfect absorber.

\section{CONCLUSION}

In this paper, we have presented a general methodology for estimation of reflection and transmission through anomalously reflecting and refracting metasurfaces. The approach is based on the use of the Huygens principle, physical optics, and the theory of diffraction gratings. This allows to find equivalent surface current densities on the Huygens surface that encloses the metasurface volume. Reflected, transmitted, and scattered fields can be then calculated integrating these currents with the corresponding Green function. Here, we used far-field approximation of these integrals, that allow derivation of simple and fully analytical formulas for the corresponding scattering patterns. These analytical results help to understand general features of scattering from metasurface panels and analyse the effects of the panel size, illumination beam width, and the wall properties. The same methodology can be used also for calculations of fields at an arbitrary points, also in the 
near zone, but in that case other approximations of the Green function should be used, or the integrals can be calculated numerically. Since we are interested in the fields outside of the source volume, the integrals are not singular, and numerical integration is simple and fast.

We hope that the models and tools developed in this paper will help to develop metasurfaces for optimization of communication channels and contribute to bridging the gap between the electromagnetic theory and design of reconfigurable metasurfaces and the communication-theory models of propagation channels in presence of such new devices.

\section{REFERENCES}

[1] C. Liaskos, S. Nie, A. Tsioliaridou, A. Pitsillides, S. Ioannidis, and I. Akyildiz, "A new wireless communication paradigm through softwarecontrolled metasurfaces," IEEE Commun. Mag., vol. 56, no. 9, pp. 162 169, 2018.

[2] M. Di Renzo, M. Debbah, D.-T. Phan-Huy, A. Zappone, M.-S Alouini, C. Yuen, V. Sciancalepore, G. C. Alexandropoulos, J. Hoydis, H. Gacanin et al., "Smart radio environments empowered by reconfigurable ai meta-surfaces: An idea whose time has come," EURASIP Journal on Wireless Communications and Networking, vol. 2019, no. 1, pp. 1-20, 2019.

[3] E. Basar, M. Di Renzo, J. De Rosny, M. Debbah, M.-S. Alouini, and R. Zhang, "Wireless communications through reconfigurable intelligent surfaces," IEEE Access, vol. 7, pp. 116753-116773, 2019.

[4] W. Tang, J. Dai, M. Chen, X. Li, Q. Cheng, S. Jin, K. Wong, and T. J. Cui, "Subject editor spotlight on programmable metasurfaces: The future of wireless?" IET Electron. Lett., vol. 55, no. 7, pp. 360-361, 2019.

[5] Q. Wu and R. Zhang, "Towards smart and reconfigurable environment: Intelligent reflecting surface aided wireless network," IEEE Commun. Mag., vol. 58, no. 1, pp. 106-112, 2020.

[6] Özdogan, E. Björnson, and E. G. Larsson, "Intelligent reflecting surfaces: Physics, propagation, and pathloss modeling," IEEE Wireless Commun. Lett., vol. 9, no. 5, pp. 581-585, 2019.

[7] C. Huang, A. Zappone, G. C. Alexandropoulos, M. Debbah, and C. Yuen, "Reconfigurable intelligent surfaces for energy efficiency in wireless communication," IEEE Trans. Wireless Commun., vol. 18, no. 8 , pp. 4157-4170, 2019.

[8] Q. Wu and R. Zhang, "Intelligent reflecting surface enhanced wireless network via joint active and passive beamforming," IEEE Trans. Wireless Commun., vol. 18, no. 11, pp. 5394-5409, 2019.

[9] P. M. D. Renzo, A. Zappone, M. Debbah, M.-S. Alouini, C. Yuen, J. D. Rosny, and S. Tretyakov, "Smart radio environments empowered by reconfigurable intelligent surfaces: How it works, state of research, and road ahead," IEEE Journal on Selected Areas in Communications, vol. 38, no. 11, pp. 2450-2525, 2020.

[10] D. Berry, R. Malech, and W. Kennedy, "The reflectarray antenna," IEEE Transactions on Antennas and Propagation, vol. 11, no. 6, pp. 645-651, 1963.

[11] J. Huang and J. A. Encinar, Reflectarray Antennas. Wiley, 2008.

[12] P. Nayeri, F. Yang, and A. Z. Elsherbeni, Reflectarray Antennas: Theory, Designs, and Applications. Wiley-IEEE Press, 2018.

[13] N. Mohammadi Estakhri and A. Alù, "Wave-front transformation with gradient metasurfaces," Phys. Rev. X, vol. 6, p. 041008, Oct 2016.

[14] A. Epstein and G. V. Eleftheriades, "Synthesis of passive lossless metasurfaces using auxiliary fields for reflectionless beam splitting and perfect reflection," Phys. Rev. Lett., vol. 117, p. 256103, Dec 2016.

[15] V. S. Asadchy, M. Albooyeh, S. N. Tcvetkova, A. Díaz-Rubio, Y. Ra'di, and S. Tretyakov, "Perfect control of reflection and refraction using spatially dispersive metasurfaces," Physical Review B, vol. 94, no. 7, p. 075142, 2016.

[16] A. Díaz-Rubio, V. Asadchy, A. Elsakka, and S. Tretyakov, "From the generalized reflection law to the realization of perfect anomalous reflectors," Science Advances, vol. 3, no. 8, p. e1602714, 2017.

[17] V. S. Asadchy, A. Wickberg, A. Díaz-Rubio, and M. Wegener, "Eliminating scattering loss in anomalously reflecting optical metasurfaces," ACS Photonics, vol. 4, no. 5, pp. 1264-1270, 2017.

[18] Y. Ra'di, D. L. Sounas, and A. Alù, "Metagratings: Beyond the limits of graded metasurfaces for wave front control," Physical Review Letters, vol. 119 , no. 6,2018
[19] D.-H. Kwon, "Lossless scalar metasurfaces for anomalous reflection based on efficient surface field optimization," IEEE Antennas and Wireless Propagation Letters, vol. 17, no. 7, pp. 1149-1152, 2018.

[20] A. Díaz-Rubio, J. Li, C. Shen, S. Cummer, and S. Tretyakov, "Power flow conformal metamirrors for engineering wave reflections," Science Advances, vol. 5, no. 2, p. eaau7288, 2019.

[21] X. Wang, A. Díaz-Rubio, and S. A. Tretyakov, "Independent control of multiple channels in metasurface devices," Phys. Rev. Applied, vol. 14, p. 024089, Aug 2020 .

[22] J. Budhu and A. Grbic, "Perfectly reflecting metasurface reflectarrays: Mutual coupling modeling between unique elements through homogenization," IEEE Transactions on Antennas and Propagation, vol. 69, no. 1, pp. 122-134, 2020.

[23] J. P. S. Wong, A. Epstein, and G. V. Eleftheriades, "Reflectionless wideangle refracting metasurfaces," IEEE Antennas and Wireless Propagation Letters, vol. 15, pp. 1293-1296, 2016.

[24] A. Epstein and G. V. Eleftheriades, "Arbitrary power-conserving field transformations with passive lossless omega-type bianisotropic metasurfaces," IEEE Transactions on Antennas and Propagation, vol. 64, no. 9, pp. 3880-3895, 2016.

[25] G. Lavigne, K. Achouri, V. S. Asadchy, S. A. Tretyakov, and C. Caloz, "Susceptibility derivation and experimental demonstration of refracting metasurfaces without spurious diffraction," IEEE Transactions on Antennas and Propagation, vol. 66, no. 3, pp. 1321-1330, 2018.

[26] M. Chen, E. Abdo-Sánchez, A. Epstein, and G. V. Eleftheriades, "Theory, design, and experimental verification of a reflectionless bianisotropic Huygens' metasurface for wide-angle refraction," Phys. Rev. B, vol. 97, p. 125433, Mar 2018.

[27] D.-H. Kwon, "Planar metasurface design for wide-angle refraction using interface field optimization," IEEE Antennas and Wireless Propagation Letters, vol. 20, no. 4, pp. 428-432, 2021.

[28] A. Diaz-Rubio and S. Tretyakov, "Macroscopic modeling of anomalously reflecting metasurfaces: Angular response and far-field scattering," IEEE Transactions on Antennas and Propagation, 2021.

[29] J. C. B. Garcia, A. Sibille, and M. Kamoun, "Reconfigurable intelligent surfaces: Bridging the gap between scattering and reflection," IEEE Journal on Selected Areas in Communications, vol. 38, no. 11, pp. 25382547, 2020.

[30] Özdogan, E. Björnson, and E. G. Larsson, "Intelligent reflecting surfaces: Physics, propagation, and pathloss modeling," IEEE Wireless Communications Letters, vol. 9, no. 5, pp. 581-585, 2020.

[31] M. Najafi, V. Jamali, R. Schober, and H. V. Poor, "Physics-based modeling and scalable optimization of large intelligent reflecting surfaces," IEEE Transactions on Communications, vol. 69, no. 4, pp. 2673-2691, 2021.

[32] D. Dardari, "Communicating with large intelligent surfaces: Fundamental limits and models," IEEE Journal on Selected Areas in Communications, vol. 38, no. 11, pp. 2526-2537, 2020.

[33] G. Gradoni and M. Di Renzo, "End-to-end mutual coupling aware communication model for reconfigurable intelligent surfaces: An electromagnetic-compliant approach based on mutual impedances," IEEE Wireless Communications Letters, vol. 10, no. 5, pp. 938-942, 2021.

[34] M. J. Mencagli, E. Martini, S. Maci, and M. Albani, "A physical optics approach to the analysis of metascreens," IEEE Access, vol. 8, pp. 162 634-162 641, 2020.

[35] F. H. Danufane, M. Di Renzo, J. De Rosny, and S. Tretyakov, "On the path-loss of reconfigurable intelligent surfaces: An approach based on green's theorem applied to vector fields," IEEE Transactions on Communications, pp. 1-1, 2021.

[36] A. Osipov and S. Tretyakov, Modern electromagnetic scattering theory with applications. John Wiley \& Sons, 2017.

[37] R.-B. Hwang, Periodic structures: mode-matching approach and applications in electromagnetic engineering. John Wiley \& Sons, 2012.

[38] X. Wang, A. Díaz-Rubio, V. S. Asadchy, G. Ptitcyn, A. A. Generalov, J. Ala-Laurinaho, and S. A. Tretyakov, "Extreme asymmetry in metasurfaces via evanescent fields engineering: Angular-asymmetric absorption," Physical Review Letters, vol. 121, no. 25, p. 256802, 2018. 